\title{
Interaction of Sedimenting Drops in a Miscible Solution -Formation of Heterogeneous Toroidal-Spiral Particles
}

\author{
Magdalena Szymusiak,Vishal Sharma, Ludwig C. Nitscheand Ying Liu* \\ Department of Chemical Engineering, University of Illinois at Chicago, Chicago, IL 60607, USA. Fax: \\ (312) 996-0808; Tel: (312) 996-8249; E-mail: liuying@uic.edu
}

\begin{abstract}
Toroidal-spiral structures form through the interaction of horizontally and vertically displaced drops sedimenting in a miscible bulk solution. These polymeric drops are then solidified into particles, which can be potentially used to encapsulate and deliver multiple active compounds on separate schedules. Sedimentation regimes and drug release were quantified.
\end{abstract}

Toroidal-spiral (TS) particles, with large surface-to-volume ratio and well-defined internal channels, were recently generated by a novel process of flash solidification of polymeric droplets during their sedimentation in a miscible solution. ${ }^{1}$ TS particles have an overall doughnut or toroidal shape and a thin spiral-shaped channel near the surfaces (Fig. 1a). Entrainment of the bulk solution within the spiral-shaped channel offered a mechanism for encapsulating active agents under benign flow conditions (low shear with only an aqueous phase). Thus, TS particles may have significant potential applications for tissue engineering and drug delivery. Previously generated toroidal or doughnut-shaped particles offer advantages compared to the spherical beads because of their larger surface to volume ratio and closer proximity of all points in the interior to the surface. ${ }^{2,3}$ However, without the feature of internal channels, drug release from the doughnut-shaped particles occurs by the same processes as for spherical beads: hindered 
diffusion through the polymeric matrix and/or degradation of the matrix. Both of these mechanisms are challenging to tune for different drugs and especially for multiple drugs with separate release schedules. By contrast, release through the well-defined nano- and microchannels of TS particles can be much more easily predicted and controlled. The main advance of this paper beyond our previous, single-drop generation of TS particles is to harness the interaction of two or more sedimenting drops to produce the intricate liquid-phase structures that are subsequently solidified. Multiple drops potentially offer the flexibility to encapsulate multiple active agents without concern for their mutual compatibility in one solution. Furthermore, the amount of encapsulation can be precisely controlled with the known volume of the drop and concentration of the active compounds. Additionally, understanding drop interaction is essential for mass production of TS particles using parallel or sequential arrays of sedimenting drops. 
a)
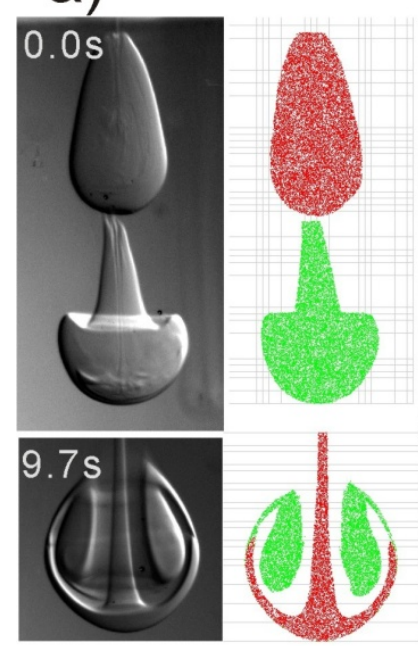

b)
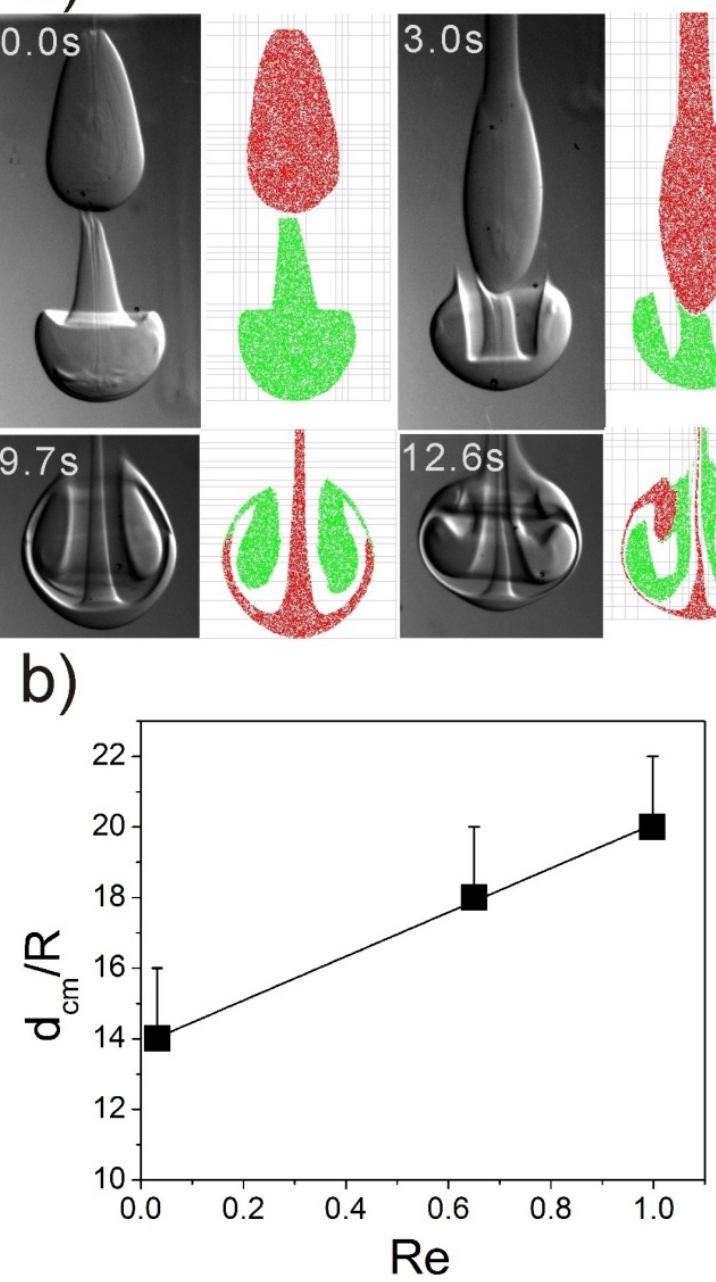
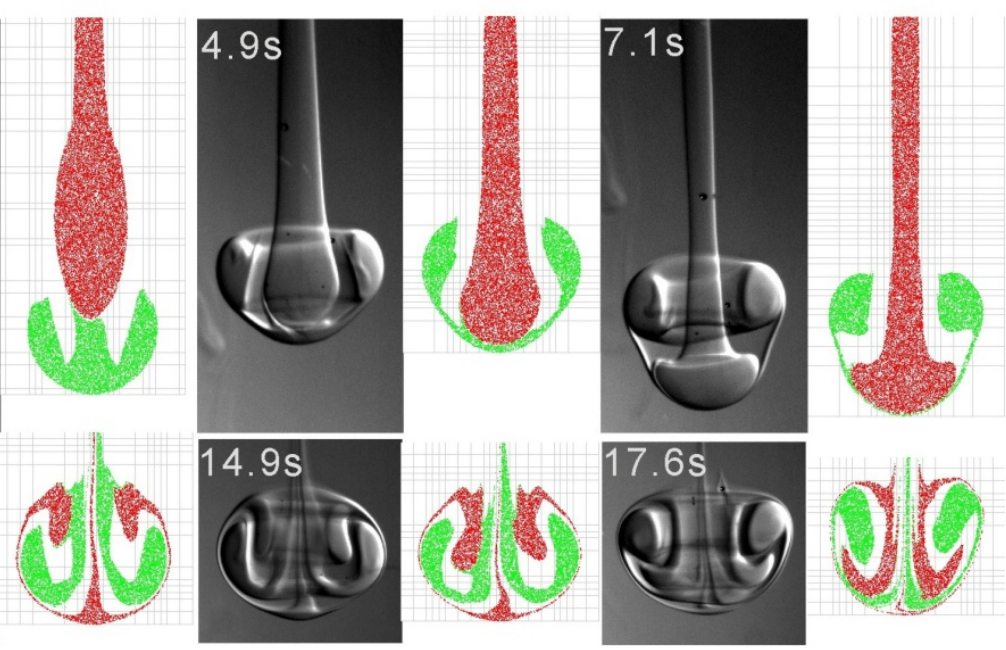

c)

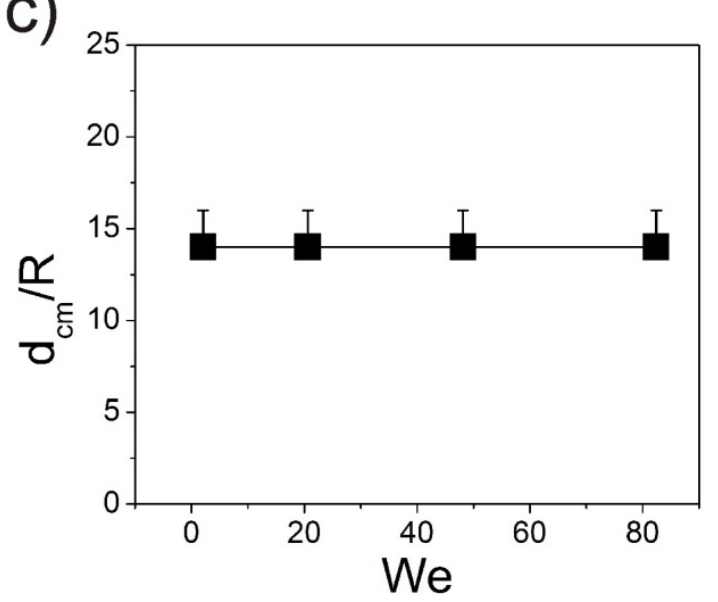

Fig. 1 (a) Time sequence of two drops sedimenting in a miscible solution - comparison of high-speedcamera images with simulations. Experimental $R e$ is 0.03 . Simulations assume zero $R e$. The time intervals were marked on the images with the unit of second (s). (b) Linear dependence of critical merging distance on Re. (c) Negligible dependence of critical merging distance on $W e$ at $R e=0.03$.

Interaction, deformation, and coalescence of viscous drops have been extensively studied to understand sedimentation and phase separation in emulsions, liquid-liquid extraction, and raindrop formation. ${ }^{4,5}$ In these systems the surrounding liquid is immiscible with the drops, and 
the strength of viscous deforming forces relative to the stabilizing influence of interfacial tension is quantified by the dimensionless Bond number. For a range of Bond numbers and lateral distances, four regimes of drop interaction have been defined for low-Reynolds-number sedimentation: separation, capture or merging, breakup, and pass-through. At finite Bond number (i.e., appreciable influence of interfacial tension) the formation of such intricate internal structures as TS channels is inhibited. ${ }^{4}$ Microfluidic techniques for encapsulating one drop within another apply to immiscible systems, ${ }^{6,7}$ and would appear less conducive to scale-up than parallel arrays of sedimenting drops. In the immiscible context, one recent $\operatorname{paper}^{8}$ describes collisions between two drops falling in air for liquid encapsulation.

The interaction of two sequential drops while sedimenting in a miscible bulk solution with various horizontal offsets was previously observed and simulated numerically. ${ }^{9}{ }^{10} \mathrm{We}$ consider systems of horizontally and vertically displaced drops with different solution compositions, elucidate the internal entrainment structures in more quantitative detail, and carry out a systematic parametric study of regimes leading to coalescence. The literature concerning vortex interaction at moderate or high Reynolds number is less relevant. ${ }^{11}$

One purpose of this paper is to describe the formation of polymeric particles with welldefined TS channels by the interaction of multiple sedimenting drops in a miscible solution prior to their photo-initiated cross-linking. By exploiting the drop dynamics and interactions, we can manipulate the structure of the particles and thereby the release kinetics of the encapsulated compounds. PEG-DA 700 (poly(ethylene glycol) diacrylate of molecular weight 700), a biocompatible polymer, was used as a model system. The properties of the polymer solution and cross-linked polymer matrix are tunable by selecting within a wide range of molecular weights and concentrations of PEG-DA. ${ }^{12-14}$ For our study, a relatively low molecular-weight PEG-DA 
was chosen so that the drop solution is Newtonian and the solidified polymer matrix has dense pores. $^{15}$ The photopolymerization conditions including the UV light intensity and the concentration of the cross-linking initiator were optimized to ensure flash solidification before drop configuration could be altered by convection.

The leading drop (in a sequential, two-drop system) or central drop(s) (in a parallel, multi-drop system) is/are at least as big as other one(s) and form(s) the main structure of the TS particle. These drops have high concentration of polymer $(83 \%)$, resulting in very dense pores that would not allow macromolecules to diffuse across the matrix. The trailing or surrounding drops are entrained to form the TS layer and can becomposed of different chemicals which need only be miscible with the bulk solution and each other.

The bulk phase is an aqueous solution with glycerol and ethanol to match the viscosity of the polymeric drop phase and to provide a suitable density difference for the desired sedimentation Reynolds number $(R e)$, which represents the ratio of inertial vs. viscous forces. As described in the Electronic Supplementary Information (ESI $\dagger$ ), a single needle or a multicapillary assembly fed by syringe pumps was used to generate polymer drops with equivalent spherical radii ranging from 700 to $2000 \mu \mathrm{m}$.

The evolutionary stages of the entrainment and deformation of the two vertically displaced sedimenting drops with various distances were observed experimentally and compared with numerical simulations, as shown in Fig.1a. The details of the simulation method is described in the (ESI $\dagger$ ). The far-field interaction explains the drop deformation at early stages: (i) the diverging stream lines below the trailing drop causes the flattening of the leading drop into an oblate shape; and (ii) the converging streamlines above the leading drop causes the trailing drop to elongate into a prolate shape. The trailing drop may catch up with 
the leading drop and pass through it, and then enmesh with it while forming the TS structure. For two sequential drops with the same $R e$ (such as drops of similar size and solution density), catching-up would eventually happen regardless of their initial separation because of this far-field interaction. As expected, a longer travel distance was required for the trailing drop to overtake the leading drop if they were initially further separated. Above a critical distance (critical merging distance, $d_{c m}$ ), no fused drop would form, because the leading drop had already bifurcated (or broken up) when the trailing one caught up with it. This critical distance depends on Re but not Weber number (We, ratio of kinetic energy over surfacetension energy during splashing implact) (Fig. $1 \mathrm{~b}$ and $1 \mathrm{c}){ }^{1}$

In making TS particles by sequential drop interactions, we usually reduce the relative size of the trailing drop to optimize the structure. In these cases, the smaller trailing drop may never catch up to the leading one (as opposed to catching up with the remains of a disintegrated leading drop, $d_{c m}$ ). This effect defines a new critical distance (critical catch-up distance, $d_{c c}$ ) for drop catch-up, which depends on the ratio of radii, ratio of excess densities, and the initial separation as predicted by simulations (Fig. 2). However, density has weaker effects on drop catch-up compared to size (Fig. S3, ESI $\dagger$ ). 
a)

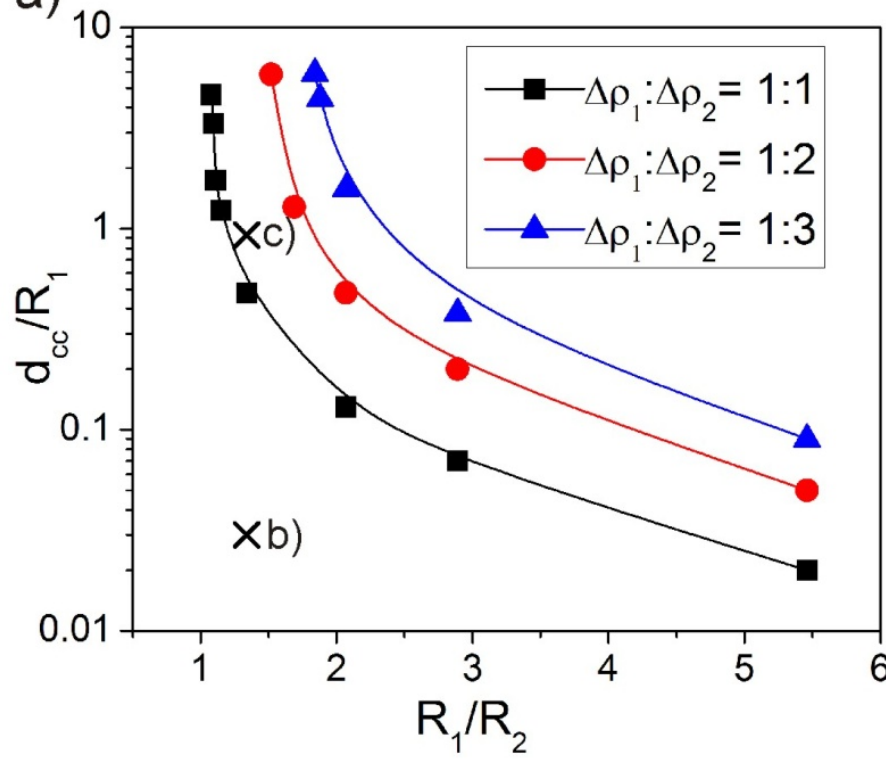

b)

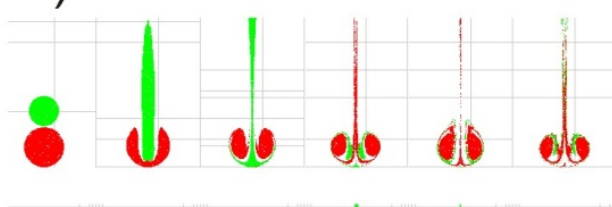

c)

Fig. 2 (a) Numerically simulated dependence of critical catch-up distance on $R_{1} / R_{2}$ and $\Delta \rho_{1} / \Delta \rho_{2}$. The subscripts 1 and 2 refer to the leading drop and the trailing drop, respectively. $R$ is the radius of the drop and $\Delta \rho$ is the density difference defined as $\Delta \rho=\rho_{\text {drop }}-\rho_{b u l k . .}$ (b) Within the critical catch-up distance $\left(\mathrm{d} / \mathrm{R}_{1}=0.03<\mathrm{d}_{\mathrm{cc}} / \mathrm{R}_{1}\right.$, at $\mathrm{R}_{1} / \mathrm{R}_{2}=1.34$ and $\left.\Delta \rho_{1} / \Delta \rho_{2}=1: 1\right)$, the trailing drop caught up with and merged into the leading one. The images are corresponding to the condition marked by $\times b$ ) in (a). (c) Beyond the critical catch-up distance $\left(\mathrm{d} / \mathrm{R}_{1}=0.93>\mathrm{d}_{\mathrm{cc}} / \mathrm{R}_{1}\right.$, at $\mathrm{R}_{1} / \mathrm{R}_{2}=1.34$ and $\left.\Delta \rho_{1} / \Delta \rho_{2}=1: 1\right)$, the two drops separated without merging. The images are corresponding to the conditions marked by $\times c)$ in $(a)$.

Making TS particles by cross linking the interacting drops also offers manufacturing flexibility by using drops of different compositions. There are two reasons why one might, for example, wish to dissolve the active agent within the trailing drop (which may contain less or no polymer) that becomes entrained inside the leading drop (of high polymer concentration). (1) The active compound may be insoluble in aqueous solution or incompatible with the polymer because of less mixing entropy of the polymer or $\chi$ parameter mismatch based on Flory-Huggins 
theory. In this case the trailing drop solution could be an organic, water-miscible solvent. (2) The active compound may be highly toxic or too expensive to be processed in bulk solutions.

A related application of differing drop compositions is demonstrated by our measurements of release kinetics for polyphosphate (with average size of 45 repeating units, $\mathrm{Mw}$ 3554) from TS particles. Polyphosphate was chosen as a model active macromolecule, because of its important role in blood coagulation and well-established characterization methods of high chemical sensitivity. Every measurement of polyphosphate release was conducted on five TS particles at similar evolutionary stages. Here the leading drop is of high concentration of PEGDA $700(83 \mathrm{wt} \%)$ to form a dense, impenetrable matrix of small pores upon cross linking. The trailing drop (which becomes entrained within the internal TS layer) contains polyphosphate along with a lower concentration of PEG-DA (0 - $40 \mathrm{wt} \%)$ that solidifies into a network with bigger pores. Meanwhile, the surrounding bulk fluid (without any PEG-DA) co-entrained to form a clear ribbon adjacent to the polymer layer in the TS channel (Fig. 3b). The polyphosphate is more likely to diffuse out along the TS pathway. Therefore, the diffusion rates of polyphosphate could be controlled by varying the porous structure of the TS layer, whichdepend on the concentration of PEG-DA in the trailing drop. At low PEG-DA concentrations $(0-10 \mathrm{wt} \%)$ in the TS layer, TS particles prolong the release of polyphosphate compared to the hemi-spherical particles (control particles), since the diffusional path length in the TS channel is longer. At relatively high PEG-DA concentrations (30 - $40 \mathrm{wt} \%$ ) in the TS layer, polyphosphate release is faster in the TS particles compared to the control, because of the unhindered diffusion through the clear ribbon (Figure 3b). 


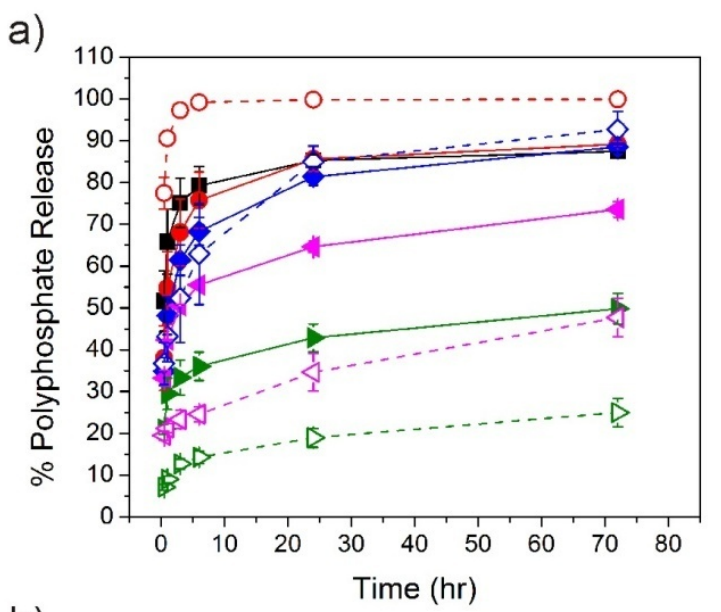

b)

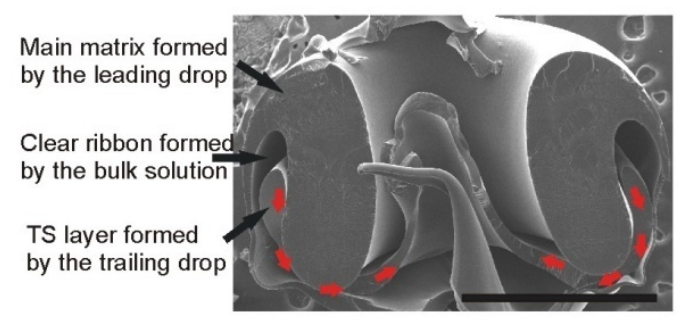

Fig. 3 (a) Release kinetics of polyphosphate from TS particles (closed symbols with solid lines) compared to the release from hemi-sphere particles (open symbols with dashed lines). Colors correspond to various PEG-DA concentrations in the TS channel (for the TS particle) or in the hemi-sphere particles: black$0 \mathrm{wt} \%$, red-10wt $\%$, blue-20wt $\%$, magneta-30wt $\%$, and green-40wt $\%$. (b) SEM images of the cross-linked TS particles. Scale bar represents $1 \mathrm{~mm}$. The red arrows indicate the release pathway of the encapsulated polyphosphates.

Another way of manipulating the release of polyphosphate is varying the length of the TS channel, which is done by solidifying the interacted drops at different stages of the shape evolution (see Fig. S4, ESI $\dagger)^{1}$ 
The interactions of two or more drops sedimenting in parallel were tested experimentally and compared with the simulations. Due to nonlinear coupling of drop deformation and sedimentation, two identical drops repelled each other and formed individual TS structures (Fig. 4a). In the case of three parallel drops, the middle drop was always pushed to a lower position and the surrounding drops were entrained into the middle one to form a fused drop with TS structure (Fig. 4b). The interaction distance $\left(d_{l}\right)$ is defined as the cumulative distancetraveled by the middle drop until the two adjacent drops begin to roll up along the outer layer formed by the middle drop (Fig. 4b at 16s). When the drops were placed further apart, the interaction distance increased linearly with the horizontal separation $\left(d_{2}\right)$ (Fig. 5). At larger $R e$, the interaction distance was shorter for the same initial separation. However, the slopes of the lines at different Re were all the same. The initial shape of the surrounding drops (immediately after injection, Fig. $4 \mathrm{~b} 0 \mathrm{~s})$ was slightly asymmetric. A test simulation considering the asymmetric shape of the surrounding drops (Fig. 5, open squares) was conducted and compared with a similar simulation using the center drop shape (Fig. 5, solid squares) for all three. The effects on the subsequent shape evolution (Fig. 4b) and interaction distance (Fig. 5) was found to be negligible. 

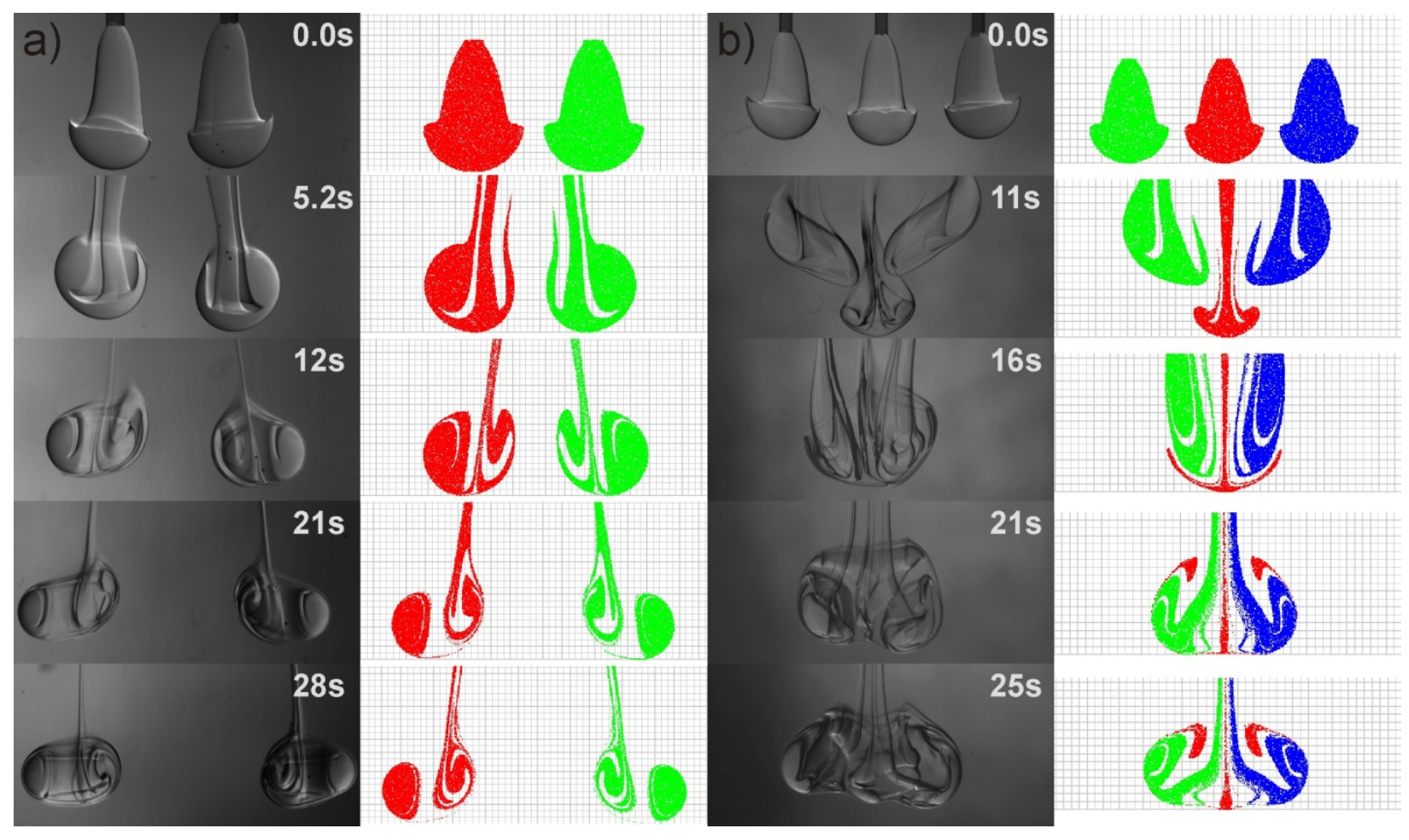

Fig. 4 Time sequence of (a) two and (b) three parallel drop sedimenting in a miscible solution comparison of high-speed-camera images with simulations. 


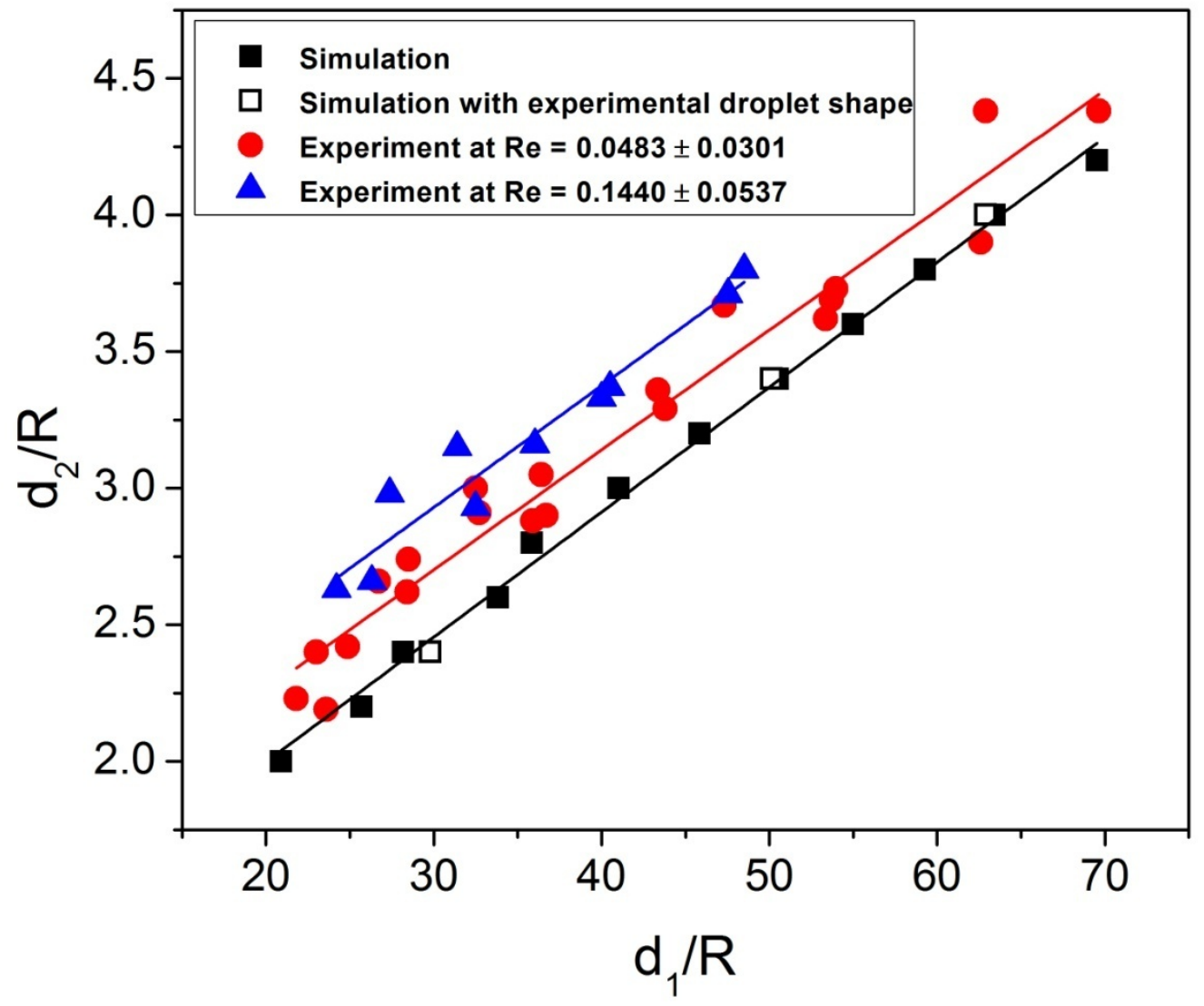

Fig. 5 Linear relation between interaction distance $\left(d_{1}\right)$ and horizontal separation $\left(d_{2}\right)$ for three-paralleldrop sedimentation at low $R e$.

For four parallel drops, the two drops at the edge merged with the two drops in the middle, forming two individual TS structures. Then, the subsequent sedimentation was similar to that of two parallel drops, which eventually repelled each other (see Fig. S5, ESI $\dagger$ ). With the simulations validated against the above experimental observations, sedimentation of close packed six and seven parallel drops have been investigated by simulation (Fig. S6 and Fig.S7, ESI†). Based upon both observations and simulations for two or more drops displaced 
horizontally, the drop-sedimentation behavior could be summarized as follows. The center drop(s) always travel(s) faster, and therefore act(s) as the leading drop(s). The surrounding drops move toward vertical alignment with the leading drop(s). For the tested separation distances, catching-up and merging of the multiple drops were nearly simultaneous, although noticeably in order of position. The two surrounding drops closer to the center one(s) entrained in first and then the next outer ones. For even number of drops, eventually the two merged drops repel each other. For odd number of drops, eventually one fused drop forms.

The results of drop interaction during parallel sedimentation can be used to design the array of injectors for mass production of the TS particles and for generating TS particles encapsulating multiple compounds dissolved in surrounding drops. To illustrate the latter, we have placed four surrounding drops (which could contain different agents), around a center polymer drop (Fig. 6) and solidified the structure.

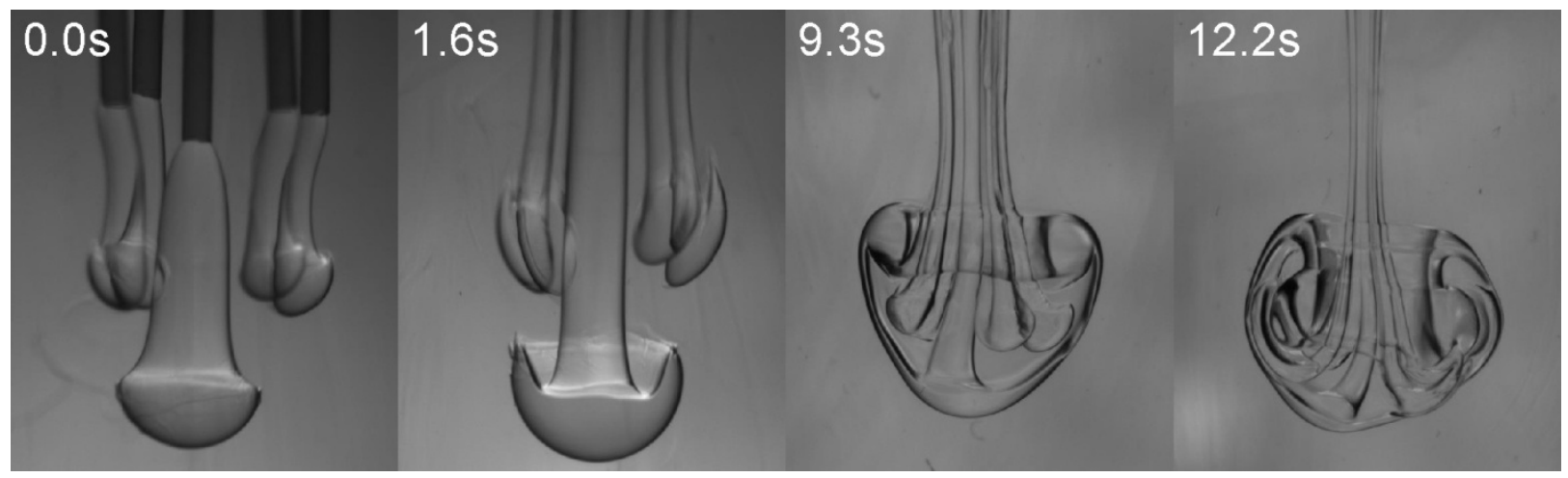

Fig. 6 Entrainment of four surrounding drops within the center drop to form a heterogeneous TS structure.

In summary, we introduced a general one-step technique for the continuous fabrication of composite polymeric particles with TS internal structures. The liquid TS structures, selfassembled by the interaction of viscous drops during sedimentation at low $R e$ in a miscible 
solution, were solidified by UV-triggered cross-linking. By our technique, multiple compounds can be encapsulated into one particle without therestriction of solution compatibility. By varying polymer concentrations in different surrounding drops, release kinetics of various compounds may be manipulated separately to reach the best synergistic effects. Currently, the particles are larger than $500 \mu \mathrm{m}$, and could be produced at the rate of at least 100 particles per hour with a single injector. The immediate biomedical applications could be through subcutaneous or post-

surgical implants. ${ }^{16}$ Moreover, our quantitative analysis of the interaction of the vertically and horizontally displaced drops could be useful for other chemical and pharmaceutical processes.

\section{Acknowledgements}

This work was supported by NSF-CBET (CBET-1039531). The authors are grateful to Professors Thomas Witten, Ka Yee Lee, Sidney Nagel, and Heinrich Jaeger at University of Chicago for the access to their MERSEC facility.

\section{References}

1 V. Sharma, M. Szymusiak, H. Shen, L. C. Nitsche and Y. Liu, Langmuir, 2012, 28, 729.

2 S. Ungphaiboon, D. Attia, G. G. d'Ayala, P. Sansongsak, F. Cellesi and N. Tirelli, Soft Matter,2010,17, 4070.

3 B. G. Wang, H. C. Shum and D. A.Weitz, ChemPhysChem,2009, 10, 641.

4 J. Kushner, M. A. Rother and R. H. Davis, J. Fluid Mech., 2001, 446, 253.

5 X. Jiang and A. J. James, J. Eng. Math, 2007, 59,99. 
6 H. S. Chen, Y. J. Zhao, J. Li, M. Guo, J. D. Wan and D. A. Weitz, and H. A. Stone, Lab Chip, 2011, 11, 2312.

7 C. Berkland, K. Kim and Daniel W. Pack, J. Controlled Release, 2001, 73, 59.

8 C. Planchette, E. Lorenceau and G. Brenn, Fluid Dynam. Mater. Process., 2011, 7, 279.

9 M. Manga and H. A. Stone, J. Fluid Mech.,1993, 256, 647.

10 G. Machu, W. Meile, L. C. Nitsche and U. Schaflinger, J. Fluid Mech.,2001, 447, 299.

11 S. Kida, M. Takaoka and F. Hussain, J. Fluid Mech.,1991, 230, 583.

12 K. Guo and C. C. Chu, J.Biomater. Sci., Polym. Ed., 2007, 18, 489.

13 M. B. Mellott, K. Searcy and M. V. Pishko, Biomaterials, 2001, 22, 929.

14 S. F. A. Hossainy and J. A. Hubbell, Biomaterials, 1994, 15, 921.

15 V. Chan, P. Zorlutuna, J. H. Jeong, H. Kong and R. Bashir, Lab Chip, 2010, 10, 2062.

16 M. A. Moses, H. Brem and R., Langer, Cancer Cell,2003,4, 337. 